\title{
RESPOSTA IMPULSIVA DE ELETRODOS DE ATERRAMENTO
}

\author{
Rafael Silva Alípio* \\ rafael.alipio@gmail.com \\ Márcio Matias Afonso* \\ marciomatias@des. cefetmg.br
}

\author{
Marco Aurélio de Oliveira Schroeder ${ }^{\dagger}$ \\ schroederdufsj.edu.br
}

Tarcísio Antônio Santos de Oliveira*

tarcisioddes.cefetmg.br

\author{
Sandro de Castro Assis ${ }^{\ddagger}$ \\ sandro.assisdcemig.com.br \\ ${ }^{*}$ Departamento de Engenharia Elétrica - DEE \\ Centro Federal de Educação Tecnológica de Minas Gerais \\ (CEFET-MG), Av. Amazonas, 7675, Nova Gameleira, CEP 30510-000, \\ Belo Horizonte, MG, Brasil \\ ${ }^{\dagger}$ Departamento de Engenharia Elétrica - DEPEL \\ Universidade Federal de São João del-Rei (UFSJ), Praça \\ Frei Orlando, 170, Centro, CEP 36307-352, \\ São João del-Rei, MG, Brasil \\ ${ }^{\ddagger}$ Companhia Energética de Minas Gerais - Cemig - PE/LS \\ Av. Barbacena, $1200-11^{\circ}$ andar - Ala A1 \\ - CEP: 30190-131, Belo Horizonte, MG, Brasil
}

\section{ABSTRACT}

Impulse response of grounding electrodes

This paper presents a series of parametric analysis of the transient behavior of horizontal grounding electrodes, typical of the transmission line counterpoises. The analyses in question consist of the relationship among grounding resistance, impulse impedance, effective length, impulse coefficient and current distribution along the electrode. The calculation of these parameters follows from the application of a grounding electromagnetic model in the frequency domain, directly derived from field equations and experimentally validated by comparison with experimental results.

KEYWORDS: Grounding, electromagnetic transients, lightning, electromagnetic modeling.

Artigo submetido em 07/12/2010 (Id.: 1230)

Revisado em 03/06/2011, 19/09/2011, 07/10/2011

Aceito sob recomendação do Editor Associado Prof. Antonio Carlos Zambroni de Souza

\section{RESUMO}

Este artigo apresenta uma série de análises paramétricas do comportamento transitório de eletrodos horizontais de aterramentos elétricos típicos dos cabos contrapeso de linhas de transmissão. As análises em causa consistem da relação entre resistência de aterramento, impedância impulsiva, comprimento efetivo, coeficiente de impulso e distribuição de corrente ao longo do eletrodo. O cálculo de tais parâmetros decorre da aplicação de uma modelagem eletromagnética de aterramentos elétricos no domínio da frequência, oriunda diretamente das equações de campo e validada experimentalmente por meio de comparação com resultados experimentais.

PALAVRAS-CHAVE: Aterramentos elétricos, transitórios eletromagnéticos, descargas atmosféricas, modelagem eletromagnética. 


\section{INTRODUÇÃO}

Quando solicitado por correntes impulsivas, advindas da incidência de descargas atmosféricas, $o$ aterramento apresenta comportamento singular e, na maior parte das situações, bastante distinto daquele observado frente a correntes de baixa frequência (Visacro, 2007). O estabelecimento de modelos confiáveis, capazes de representar adequadamente tal comportamento é de fundamental importância para avaliação do desempenho do aterramento e, também, do sistema aterrado (Alípio et alli, 2011).

A modelagem de aterramentos para fenômenos impulsivos tem sido tema de estudo de diversos grupos de pesquisa internacionais nas últimas décadas (Verma e Mukhedkar, 1980; Meliopoulos e Moharam, 1983; Mazzetti e Veca, 1983; Velazquez e Mukhedkar, 1984; Papalexopoulos e Meliopoulos, 1987; Ramamoorty et alli, 1989; Grcev e Dawalibi, 1990; Dawalibi e Selby, 1993; Grcev, 1996; Olsen e Willis, 1996; Geri, 1999; Otero et alli, 1999; Liu et alli, 2001; Lorentzou et alli, 2003; Liu et alli, 2005; He et alli, 2005; Zeng et alli, 2008).

No caso do Brasil, que apresenta algumas características peculiares tais como valor médio de resistividade elevado e alta densidade de incidências de descargas atmosféricas para terra, o tema apresenta particular importância (Cherchiglia et alli, 1997). Em termos de trabalhos nacionais pode-se citar aqueles pioneiros desenvolvidos por Visacro (1992) e Visacro e Portela (1992) e outros que se seguiram a esses como Soares (1996), Portela (1997), Miranda (2003), Rodrigues (2004), Nogueira (2006), Salari (2006), Salari e Portela (2007), Visacro et alli (1997) e Alípio (2008a).

Apesar das importantes contribuições dos trabalhos citados, observa-se a ausência de publicação que apresente de forma organizada uma análise paramétrica consistente do comportamento impulsivo do aterramento elétrico e uma comparação desse comportamento com aquele típico de solicitações lentas. O objetivo deste trabalho é apresentar tais análises, com base na aplicação de uma modelagem rigorosa, e com foco nas grandezas mais relevantes para a avaliação do comportamento dinâmico e em regime permanente do aterramento. Por simplicidade, analisam-se apenas eletrodos horizontais.

Na seção 2 deste artigo, o modelo adotado nas simulações é descrito sucintamente. Na seção 3 são definidos parâmetros para caracterização do comportamento dinâmico e em regime permanente do aterramento. Na seção 4 apresentamse os resultados e análises do comportamento impulsivo da seguinte forma: 4.1-Resultados da resistência de aterramento, da impedância impulsiva e do coeficiente de impulso, bem como da relação dessas grandezas com o comprimento efetivo do aterramento; 4.2-Resultados da impedância tran- sitória e da distribuição instantânea de corrente nos eletrodos e da relação dessas grandezas com o comportamento dinâmico e em regime permanente do aterramento e 4.3Orientações de cunho prático no projeto de aterramentos para fenômenos impulsivos com base nos resultados numéricos apresentados e na experiência dos autores. Na seção 5 são feitos os comentários finais.

\section{MODELO MATEMÁTICO}

Aplica-se neste trabalho um modelo para simulação do aterramento elétrico baseado na aplicação direta das equações de campo no domínio da frequência (Alípio et alli, 2011). Cada eletrodo é considerado um condutor cilíndrico, que, quando excitado, constitui-se fonte de corrente de dois tipos (ver Figura 1): i) longitudinal $\left(\mathrm{I}_{L}\right)$, ao longo do condutor e ii) transversal $\left(\mathrm{I}_{T}\right)$, que dele dispersa para o meio circundante. Essa idéia foi originalmente proposta por Visacro (1992) e, posteriormente, expandida por Visacro e Soares (2005) para modelagem de condutores genéricos solicitados por descargas atmosféricas, através de elementos condutores cilíndricos. Mais tarde, Alípio (2008a) propôs uma nova solução para as equações resultantes do modelo baseada na aplicação do Método dos Momentos (MoM) (Harrington, 1993). Também, nessa solução proposta, o efeito da interface solo-ar foi incluído mediante aplicações do método das imagens complexas, conforme proposto por Schroeder (2001), que adota coeficientes complexos para as imagens, em função das características eletromagnéticas de cada meio e da frequência sob análise. Os detalhes da aplicação do MoM, inclusão de imagens complexas e validação da metodologia proposta com resultados de medição do comportamento impulsivo de eletrodos de aterramento, podem ser encontrados em publicação recente de alguns dos autores deste trabalho (Alípio et alli, 2011). Apresenta-se a seguir, portanto, apenas uma descrição sucinta das idéias do modelo. Outras informações podem ser obtidas nas referências Schroeder (2001), Visacro e Soares (2005), Alípio (2008a) ou, originalmente, em Visacro (1992).

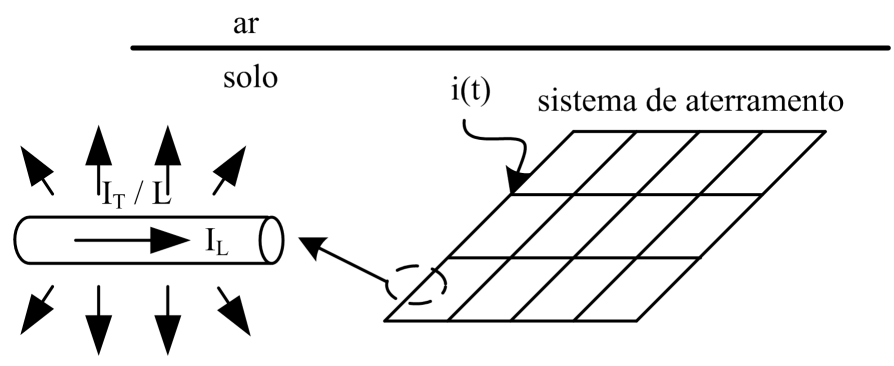

Figura 1: Esboço das fontes de corrente em cada eletrodo.

Sejam as fontes de corrente $\mathrm{I}_{T}$ e $\mathrm{I}_{L}$ associadas a um eletrodo de aterramento. A fonte de corrente transversal, devido ao 
fato de possuir natureza divergente, apresenta efeito elétrico, mas não magnético. A cada fonte de corrente transversal está associado um campo elétrico de natureza conservativa. Esse campo gera elevação de potencial $\mathrm{V}$ em relação ao infinito em pontos genéricos no meio em que o eletrodo se encontra inserido, inclusive nos demais eletrodos e nele mesmo. Por outro lado, a fonte de corrente longitudinal, devido ao fato de possuir natureza solenoidal, apresenta efeito eletromagnético. A cada fonte de corrente longitudinal está associado um campo magnético, cuja variação no tempo gera um campo elétrico de natureza não-conservativa. $\mathrm{O}$ efeito deste campo elétrico solenoidal se traduz na força eletromotriz induzida $\Delta \mathrm{V}$ em outros eletrodos e, inclusive, no próprio eletrodo fonte. Pela aplicação do MoM, o sistema de aterramento é discretizado em $\mathrm{N}$ elementos e dois sistemas matriciais são estabelecidos: i) $\mathbf{V}=\mathbf{Z}_{T} \mathbf{I}_{T}$ e ii) $\Delta \mathbf{V}=\mathbf{Z}_{L} \mathbf{I}_{L}$. O primeiro relaciona o vetor $\mathbf{V}$ dos potenciais médios em cada elemento e o vetor $\mathbf{I}_{T}$ das correntes que dispersam de cada um, por meio da matriz $\mathbf{Z}_{T}$ de impedâncias transversais, que quantifica os acoplamentos condutivo e capacitivo entre os $\mathrm{N}$ elementos. O segundo relaciona o vetor $\Delta \mathbf{V}$ das quedas de tensão em cada elemento e o vetor $\mathbf{I}_{L}$ das correntes ao longo de cada um, por meio da matriz $\mathbf{Z}_{L}$ de impedâncias longitudinais, que quantifica os acoplamentos resistivo e indutivo entre os $\mathrm{N}$ elementos. No cálculo dos elementos das matrizes $\mathbf{Z}_{T}$ e $\mathbf{Z}_{L}$, o efeito da interface solo-ar é levado em consideração pelo método das imagens complexas. O estabelecimento de relações entre $\mathbf{V}$ e $\Delta \mathbf{V}$ com os potenciais nodais $\mathbf{V}_{N}$ e entre $\mathbf{I}_{T}$ e $\mathbf{I}_{L}$ com as correntes injetadas nos nós $\mathbf{I}_{N}$ permite acoplar os dois sistemas de equações anteriormente descritos em um único da forma:

$$
\mathrm{W} \cdot \mathrm{V}_{\mathrm{N}}=\mathrm{I}_{\mathrm{N}}
$$

em que $\mathbf{W}$ é uma matriz determinada a partir de $\mathbf{Z}_{T}$ e $\mathbf{Z}_{L}, \mathbf{V}_{N}$ é o vetor de potenciais nodais (em relação ao terra remoto) e $\mathbf{I}_{N}$ é o vetor de correntes injetadas nos nós.

Salienta-se que a equação matricial (1) estabelece relações entre fasores de corrente e potencial nodais, para uma frequência específica. A determinação da resposta em frequência do aterramento é obtida solucionando-se o sistema (1) ao longo do espectro de interesse. Resultados no domínio do tempo são obtidos após a aplicação da transformada inversa de Fourier.

\section{CARACTERIZAÇÃO DO COMPORTA- MENTO TRANSITÓRIO DO ATERRA- MENTO ELÉTRICO}

A definição de parâmetros que permitam a caracterização do comportamento transitório do aterramento é essencial para avaliação de seu desempenho frente a correntes impulsivas. Neste trabalho utilizam-se parâmetros e definições usualmente empregados e já consolidados em trabalhos clássicos da área (Mazzeti e Veca, 1983; Visacro, 2002; Visacro, 2007; Grcev, 2009). Entende-se, ainda, que um dos principais objetivos na definição de quantidades que caracterizam o comportamento transitório do aterramento é a distinção entre o desempenho impulsivo e em baixa frequência. Segundo essa filosofia adota-se, neste trabalho, as definições destacadas a seguir.

Basicamente, o desempenho de aterramento em baixa frequência, além dos potenciais no nível do solo, é determinado pelo parâmetro resistência de aterramento $\mathrm{R}$, definida como (Visacro, 2002):

$$
\mathrm{R}=\frac{\mathrm{V}}{\mathrm{I}}
$$

onde $\mathrm{V}$ é a elevação de potencial desenvolvida no ponto de injeção de corrente em relação ao terra remoto e I é a corrente injetada.

$\mathrm{Na}$ caracterização do comportamento transitório, seja uma onda de corrente impulsiva $i(t)$ injetada no aterramento, que resulta em uma elevação de potencial $\mathrm{v}(\mathrm{t})$ no ponto de injeção. A impedância impulsiva $Z_{P}$ é definida como (Visacro, 2007; Grcev, 2009):

$$
\mathrm{Z}_{\mathrm{p}}=\frac{\mathrm{V}_{\mathrm{p}}}{\mathrm{I}_{\mathrm{p}}}
$$

onde $\mathrm{V}_{P}$ corresponde ao valor de pico de $\mathrm{v}(\mathrm{t})$ e $\mathrm{I}_{P}$ corresponde ao valor de pico de $\mathrm{i}(\mathrm{t})$.

O coeficiente de impulso A é um parâmetro útil na comparação entre o comportamento impulsivo e em baixa frequência do aterramento. Ele é definido como a razão entre a impedância impulsiva e a resistência de aterramento, ou seja, (Visacro, 2007, Grcev, 2009):

$$
\mathrm{A}=\frac{\mathrm{Z}_{\mathrm{p}}}{\mathrm{R}}
$$

sendo que valores de A inferiores a unidade indicam desempenho impulsivo do aterramento "superior" ao seu desempenho frente a solicitações lentas e valores de A superiores a unidade indicam desempenho impulsivo "inferior".

Outro parâmetro adotado para caracterização da resposta dinâmica do aterramento é a impedância transitória $\mathrm{z}(\mathrm{t})$, definida como (Visacro, 2007, Grcev, 2009): 


$$
\mathrm{z}(\mathrm{t})=\frac{\mathrm{v}(\mathrm{t})}{\mathrm{i}(\mathrm{t})}
$$

Tal impedância apresenta variação rápida durante o período inicial do transitório e, após alguns microsegundos, tende ao valor da resistência de aterramento R. Essa constatação motivou, em um trabalho dos autores (Alípio et alli, 2009a), a definição de dois períodos distintos da resposta transitória do aterramento: 1) período dinâmico (PD) e 2) período permanente (PP). No primeiro período, os efeitos associados a fenômenos de alta frequência, nomeadamente os efeitos reativos e de propagação, são acentuados. No segundo, tais efeitos são pouco significativos e o comportamento do aterramento é caracterizado pelo parâmetro de baixa frequência R. Apenas como referência, a Figura 2 apresenta um gráfico de $\mathrm{z}(\mathrm{t})$ para um eletrodo horizontal de aterramento de $30 \mathrm{~m}$, enterrado a $0,5 \mathrm{~m}$ de profundidade em um solo de resistividade de $500 \Omega$.m e submetido a uma corrente impulsiva, com tempos de frente e de cauda, respectivamente, de $1,2 \mu \mathrm{s}$ e de $50 \mu$ s. Os períodos PD e PP indicados na figura podem variar de acordo com o comprimento do eletrodo, resistividade do solo e onda de corrente injetada. De acordo com Alípio et alli (2009a) a duração do PD é da ordem de até alguns microsegundos e aumenta com o comprimento do eletrodo, condutividade do solo e tempo de frente da onda injetada. Pesquisas paralelas desenvolvidas por Grcev (2009) indicam conclusões neste mesmo sentido.

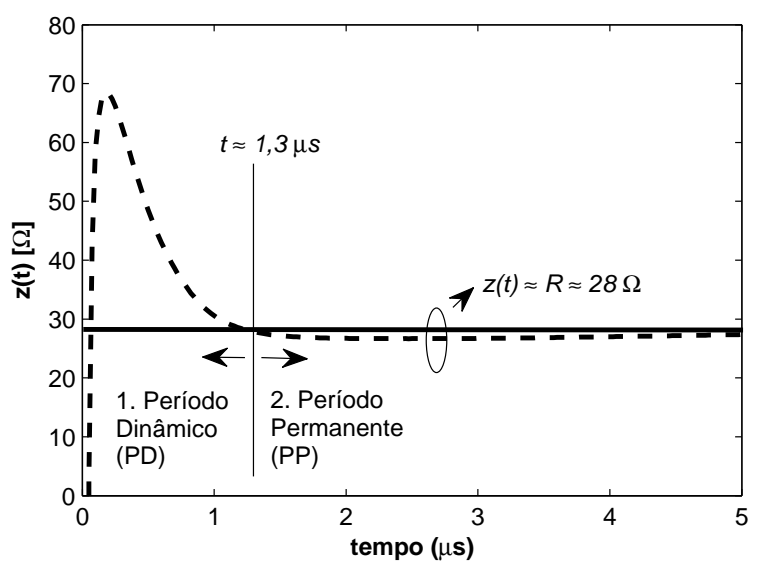

Figura 2: Impedância transitória de aterramento de eletrodo horizontal de $30 \mathrm{~m}$, enterrado a $0,5 \mathrm{~m}$ de profundidade, imerso em solo de $500 \Omega$.m

Quando uma corrente impulsiva é injetada no sistema de aterramento, a onda eletromagnética associada ao surto se propaga ao longo do eletrodo. A propagação da onda eletromagnética apresenta dois fenômenos associados: atenuação (em decorrência das perdas no solo) e distorção da onda (Visacro, 2007). O primeiro constitui-se no decréscimo da amplitude da onda de corrente ao longo do eletrodo. O segundo representa a deformação da onda à medida que se propaga e corresponde fisicamente às diferentes velocidades que cada componente de frequência possui, não apresentando assim uma propagação uniforme como um todo. A atenuação aumenta com a frequência e com a condutividade do solo (Visacro, 2007). Outros fatores também contribuem com a atenuação, como por exemplo, o fenômeno de ionização do solo (não considerados neste trabalho). Em resumo, a onda de corrente que se propaga ao longo do eletrodo de aterramento tem sua amplitude atenuada e sofre deformação, que ocasiona um aumento do tempo de frente ao longo da direção de propagação.

Tendo em conta os efeitos de propagação acentuados ao longo do PD, a corrente que dispersa do eletrodo de aterramento apresenta uma distribuição não uniforme ao longo do mesmo, como consequência direta do fenômeno de atenuação (Visacro, 2007). Dessas considerações deriva-se o importante conceito de comprimento efetivo ( $(\mathrm{ef})$, que corresponde a um comprimento limite do eletrodo de aterramento. Eletrodos com comprimento superior a esse valor limite não implicam na redução da impedância impulsiva. Este comportamento é explicado pelo fato de que além deste limiar, os componentes de alta frequência das correntes (associadas à frente da onda) estão tão atenuados que, a despeito da disponibilidade de comprimento adicional do eletrodo, praticamente não mais contribuem para dispersão de corrente para o solo (Visacro, 2007).

\section{RESULTADOS}

Os resultados de simulação apresentados nesta seção referem-se a eletrodos horizontais de raio $1 \mathrm{~cm}$, enterrados a $0,5 \mathrm{~m}$ de profundidade no solo e comprimento dentro da faixa de 5 a $90 \mathrm{~m}$. Consideram-se solos de resistividade de 100,500 e $1000 \Omega . m$ e, em todos os casos, adota-se um valor médio de permissividade relativa $\epsilon_{r}=15$. A variação dos parâmetros elétricos do solo com a frequência não foi considerada, segundo uma postura conservadora e devido à dificuldade de estabelecimento de formulações generalizadas para se ter em conta tal efeito (Alípio et alli 2009b; Pedrosa et alli 2009; Pedrosa, 2010; Pedrosa et alli 2010a, 2010b e 2010c). Os efeitos da ionização do solo não foram incluídos nas simulações, também de acordo com uma postura conservadora (Visacro, 2007; Salari e Portela, 2008).

Os eletrodos foram excitados por uma onda do tipo dupla exponencial $1,2 / 50 \mu \mathrm{s}$ (ou impulso atmosférico), tipicamente adotada em ensaios de impulso atmosférico (NBR 6939, 2000). É de conhecimento dos autores deste trabalho que esta onda não representa a forma de uma onda real de uma descarga atmosférica (Visacro, 2005). Há dois aspectos principais entre as curvas reais de descarga e a dupla exponen- 
cial. Primeiramente, a natureza côncava da frente de onda de corrente da descarga real nos instantes inicias não é contemplada pela dupla exponencial (Visacro, 2005). Por outro lado, a dupla exponencial tem sua derivada máxima justamente nesses instantes iniciais, enquanto na onda real, a derivada máxima ocorre próximo ao pico. No entanto, a despeito de sua forma pouco representativa, a onda dupla exponencial apresenta um espectro associado, que abrange as frequências típicas de transitórios oriundos da incidência de descargas atmosféricas. Dessa maneira, em termos de avaliação geral do comportamento impulsivo do aterramento, a utilização dessa onda parece adequada.

\subsection{Comprimento efetivo e comporta- mento impulsivo}

A Figura 3 apresenta os resultados de simulação da impedância impulsiva $Z_{p}$ e resistência de aterramento R de eletrodos horizontais para comprimento $\mathrm{L}$ dentro da faixa de 5 a $90 \mathrm{~m}$. São considerados três valores de resistividade: 100 , 500 e $1000 \Omega . m$.

De acordo com a Figura 3, a resistência de aterramento apresenta valores elevados para solos mais resistivos e diminui com o aumento do comprimento do eletrodo. As curvas de impedância impulsiva possuem tendência similar e indicam valores altos de $Z_{p}$ para solos de baixa condutividade e reduções de tais valores para acréscimos no comprimento. Todavia, a partir de determinada extensão de eletrodo, $\mathrm{Z}_{p}$ permanece constante, enquanto $R$ continua a decrescer. Assim, o aumento das dimensões do aterramento somente é efetivo na redução da impedância impulsiva até determinado comprimento, definido como comprimento efetivo $\ell$ ef. Ainda de acordo com a figura, pode-se inferir que $\ell$ ef decresce com o aumento da condutividade do solo. Tal comportamento está associado aos efeitos de propagação, tendo em vista que a atenuação se acentua com o crescimento da condutividade do meio. Tendo em conta as análises anteriores e a as curvas da Figura 3 estimam-se os valores de $\ell$ ef como 10,18 e $26 \mathrm{~m}$, para os solos de resistividade 100,500 e $1000 \Omega . m$, respectivamente.

Uma implicação prática imediata do comprimento efetivo é que ele determina a extensão do aterramento, a partir da qual o valor de pico da sobretensão resultante no ponto de injeção é independente do tamanho do sistema de aterramento. A Figura 4 apresenta as curvas de elevação de potencial no ponto de injeção para eletrodos de diferentes comprimentos e solo de resistividade $500 \Omega . \mathrm{m}$. Claro está que eletrodos com comprimento além do $\ell$ ef não são efetivos na redução do pico de tensão resultante. No entanto, eletrodos de extensão superior ao $\ell$ ef apresentam melhor desempenho na cauda da onda, período que é caracterizado basicamente pela resistência em baixa frequência.

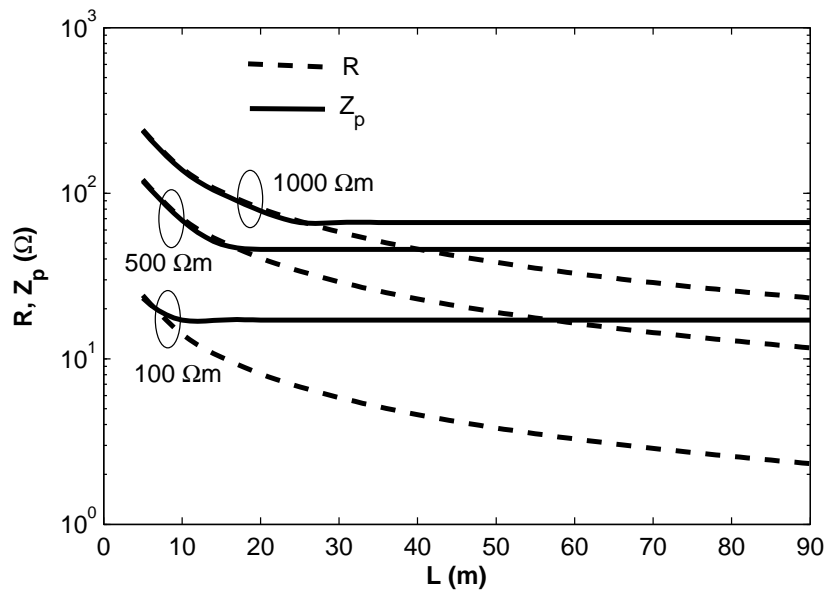

Figura 3: Resistência de aterramento $\mathrm{R}$ e impedância impulsiva $Z_{p}$ em função do comprimento do eletrodo, imerso em solos de resistividade de 100, 500 e $1000 \Omega . m$

A Figura 5 apresenta o coeficiente de impulso em função do comprimento dos eletrodos, para resistividades de $100,500 \mathrm{e}$

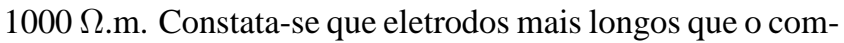
primento efetivo apresentam desempenho impulsivo inferior $(A>1)$. Por outro lado, para extensão igual ou inferior ao $\ell$ ef o desempenho impulsivo é basicamente igual ao desempenho do aterramento frente a solicitações mais lentas. Também, é importante comentar que o coeficiente de impulso não deve ser analisado individualmente, uma vez que valores elevados de A não implicam necessariamente em baixo desempenho do aterramento. Eles podem ser, por exemplo, resultado de um reduzido valor de R. Assim, para avaliação completa da informação física associada ao coeficiente de impulso, o valor da resistência em baixa frequência deve ser também analisado (Grcev, 2009). Obviamente, elevados valores de A indicam desempenho impulsivo pouco satisfatório em relação à resposta do aterramento em baixa frequência.

\subsection{Distribuição de corrente no aterra- mento}

Considera-se nesta seção um eletrodo de 40 m, excitado por um impulso atmosférico, inserido em solos de resistividade

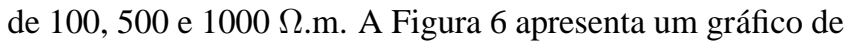
$\mathrm{z}(\mathrm{t})$ para os casos avaliados. Dela pode-se estimar a duração do PD em cerca de 1,2 e $10 \mu$ s para o eletrodo imerso em solo de 1000, 500 e $100 \Omega$.m, respectivamente. Após esses instantes de tempo o valor de $\mathrm{z}(\mathrm{t})$ tende a $\mathrm{R}$, o que caracteriza o PP da resposta do aterramento.

A Figura 7 apresenta a "distribuição instantânea" de corrente dispersa para o solo ao longo do eletrodo para 6 instantes de tempo $(0,25 ; 0,5 ; 1 ; 5,10$ e $50 \mu$ s) e (a) $\rho=100 \Omega$.m, 
(b) $\rho=500 \Omega . \mathrm{m}$ e (c) $\rho=1000 \Omega$.m. Como referência, tendo em conta uma velocidade média de propagação, o tempo de trânsito no eletrodo é de cerca de 0,$945 ; 0,522$ e $0,459 \mu \mathrm{s}$, respectivamente, para os solos de resistividade $100,500 \mathrm{e}$ 1000 S.m.

Da análise dos perfis de corrente ilustrados na Figura 7 podese distinguir dois comportamentos distintos dominantes.

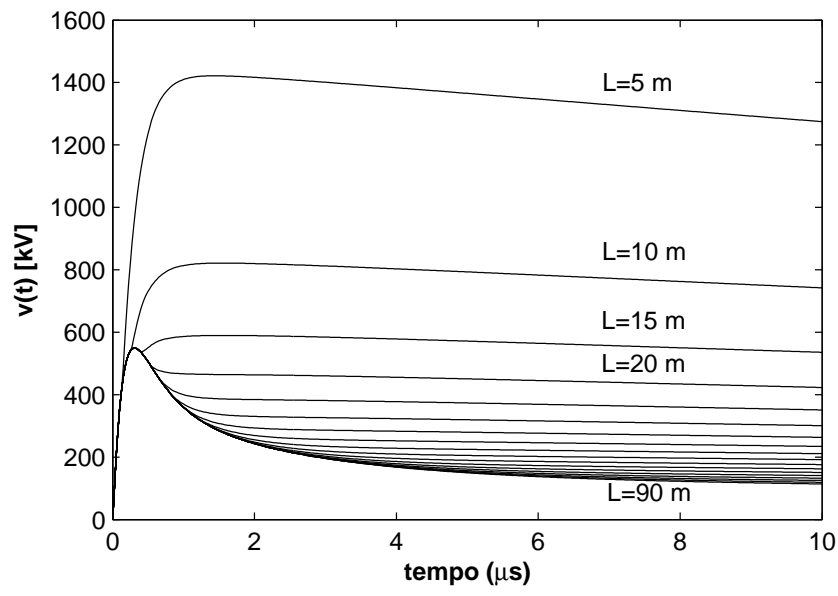

Figura 4: Elevação de potencial no ponto de injeção para diferentes comprimentos de eletrodos e solo de $500 \Omega . m$

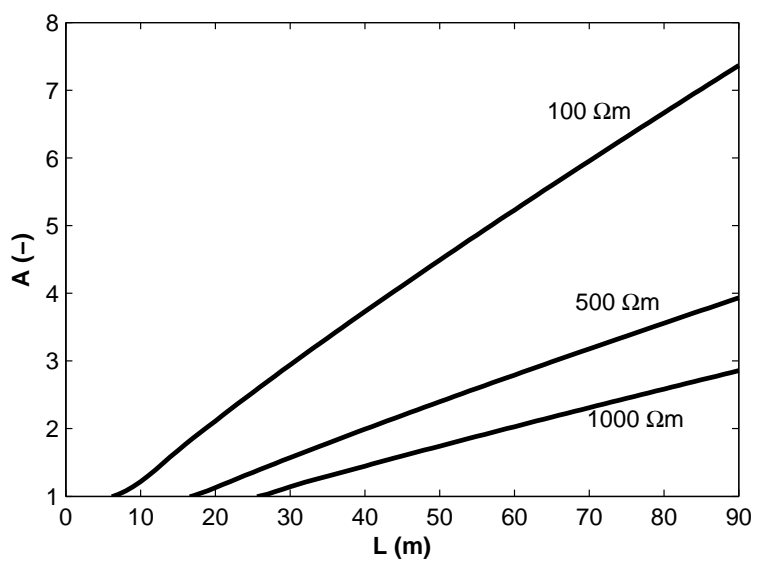

Figura 5: Coeficiente de impulso A em função do comprimento do eletrodo, imerso em solos de resistividade de 100, 500 e $1000 \Omega . m$

O primeiro está associado àqueles instantes inferiores ao tempo total do PD e caracteriza-se por um perfil de corrente não uniforme, com nítida redução da densidade de corrente de dispersão ao longo do eletrodo. Neste caso, a maior parte da corrente é dispersa em pontos próximos ao de injeção de corrente. Por outro lado, em relação a esses pontos, a corrente dispersa do eletrodo para o solo nas extensões além do

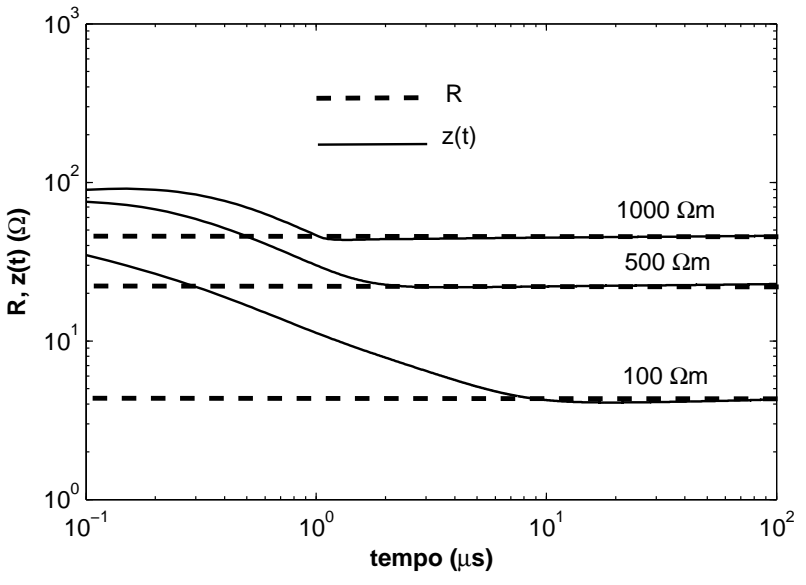

Figura 6: Resistência de aterramento $\mathrm{R}$ e impedância transitória $\mathrm{z}(\mathrm{t})$ para eletrodo de $40 \mathrm{~m}$, imerso em solos de resistividade de 100,500 e $1000 \Omega . m$

comprimento efetivo é bastante reduzida. Adicionalmente, verifica-se que os níveis de corrente injetada no solo nas vizinhanças do ponto de injeção são superiores para solos mais condutivos. Tal comportamento é prontamente compreendido tendo em conta que a velocidade de propagação é tanto menor quanto maior é a condutividade do solo. Assim, para uma mesma corrente injetada no aterramento, parcelas superiores dela são dispersas do eletrodo nas faixas iniciais de sua extensão para solos de maior condutividade em relação àqueles mais resistivos.

O segundo comportamento dominante das curvas da Figura 7 está relacionado àqueles instantes de tempo associados ao PP (ou instantes de tempo mais avançados do PD) e caracterizase por um perfil de corrente típico de solicitações de baixa frequência. Neste caso, há dois picos de corrente nas extremidades do eletrodo e uma distribuição basicamente uniforme da corrente de dispersão ao longo do restante do condutor. Vale chamar atenção ao fato de que durante o PP toda a extensão do aterramento é "efetivamente" utilizada na dispersão de corrente.

Neste ponto é importante destacar que o comprimento efetivo não deve ser interpretado como aquele suficiente para dissipar integralmente a corrente da descarga. Por outro lado, de acordo com os resultados da Figura 7, para aqueles instantes inferiores ao tempo total do PD, o lef é uma boa referência da extensão do eletrodo que é efetivamente utilizada na dispersão de corrente para o solo. Portanto, das análises anteriores pode-se inferir, que durante o PD uma extensão limitada do aterramento, associada ao comprimento efetivo, é utilizada na dissipação do impulso e é efetiva na redução do pico da elevação de potencial no ponto de injeção de corrente. $\mathrm{O}$ PD, de acordo com a Figura 6, dura apenas alguns micro- 
segundos, com limite de cerca de dez microsegundos para solos de condutividade mais elevada. Após esse período, até o fim do impulso atmosférico, ao longo do PP, a distribuição de corrente é similar àquelas típicas de solicitações de baixa frequência e toda a extensão do aterramento é efetivamente utilizada na dispersão da corrente de descarga.

\subsection{Aspectos de aplicação}

A partir das análises apresentadas nas subseções anteriores, algumas recomendações e comentários de ordem aplicada podem ser estabelecidos.

Um primeiro aspecto básico é que o conceito de comprimento efetivo diz respeito exclusivamente a eventos de frequência elevada. Portanto, não faz sentido quantificar tal parâmetro em solicitações de baixa frequência como, por exemplo, os curtos-circuitos. Ainda, deve-se salientar que, mesmo na análise de transitórios, o efeito associado ao comprimento efetivo somente é percebido em um curto período de tempo, definido neste trabalho como período dinâmico.

Outro aspecto relevante é que o comprimento efetivo não é um parâmetro constante para determinada configuração de aterramento em um solo específico. Assim, são observadas variações no valor do $\ell$ ef em função da onda de corrente, essencialmente do tempo de frente. Como referência, a partir dos resultados das subseções anteriores e de outros não incluídos neste trabalho, para solos de resistividade de 100, $500,1000,2400,3000$ e $5000 \Omega . m$, o comprimento efetivo é da ordem de $10,18,26,46,54$ e $82 \mathrm{~m}$, respectivamente, considerando uma onda de frente rápida $(1,2 / 50 \mu \mathrm{s})$. No caso de uma onda mais lenta, com tempo de frente da ordem de $3 \mu$ s, por exemplo, espera-se um aumento do valor de $\ell$ ef. Todavia, em proteção contra descargas atmosféricas, recomenda-se a utilização dos valores citados anteriormente. Tal fato é justificado uma vez que, segundo resultados da subseção anterior, a corrente dispersa para o solo de pontos próximos ao comprimento efetivo ou a partir dele é bastante reduzida. Portanto, é prática pouco econômica e eficiente adotar comprimentos de eletrodo próximos ao comprimento efetivo para ondas mais lentas. No caso de ondas com frente relativamente mais lenta (por exemplo, uma onda $8 / 20 \mu \mathrm{s}$ ), o comprimento efetivo pode atingir valores bastante elevados, particularmente quando são considerados solos mais resistivos. Nessa situação o parâmetro lef apresenta influência moderada, uma vez que, em projetos reais, tal comprimento dificilmente será atingido, com exceção de solos de baixa resistividade (da ordem de $100 \Omega$.m ou inferior).

Também é oportuno destacar, que há diferença fundamental entre a resistência e a impedância impulsiva de aterramento. A primeira é uma representação adequada do aterramento apenas em baixa frequência, enquanto a segunda é a mais adequada para avaliação da resposta dos eletrodos frente a descargas atmosféricas. $\mathrm{O}$ aumento do comprimento do eletrodo implica na redução da resistência de aterramento. Todavia, no caso de transitórios eletromagnéticos de alta frequência esse aumento do comprimento pode não refletir diretamente na redução da impedância impulsiva. Atingido o comprimento efetivo do eletrodo de aterramento, posterior aumento de sua extensão se traduz na redução de $R$, mas não de $Z_{p}$. A utilização de longos eletrodos, que possuem uma baixa resistência de aterramento, pode gerar a falsa expectativa de uma correspondente baixa impedância. Recomendase, portanto, atenção especial naqueles casos em que o comportamento impulsivo do aterramento é estimado a partir de seu comportamento em baixa frequência. São exemplos típicos dessa prática o usual critério de $20 \Omega$ para resistência de pé de torre (recomendação para fins de desempenho de linhas de transmissão frente a descargas atmosféricas) e a sugestão de cerca de $10 \Omega$ para resistência de aterramento de sistemas de proteção contra descargas atmosféricas (SPDA). Uma regra útil, segundo uma abordagem conservadora, é que o valor da resistência de aterramento é uma boa estimativa da impedância impulsiva naqueles casos em que o comprimento efetivo não é ultrapassado.

Em aplicações envolvendo proteção contra descargas atmosféricas, principalmente naquelas em que um baixo valor de impedância é requerido, é de fundamental importância a definição adequada do(s) ponto(s) de injeção de corrente.

Apesar da resistência de aterramento não depender da posição de conexão do sistema elétrico ao aterramento, a impedância impulsiva pode apresentar variações significativas de acordo com o ponto de injeção de corrente. Tais variações estão diretamente associadas ao conceito de comprimento efetivo, conforme ilustra a Figura 8. Para o eletrodo horizontal, a conexão no ponto A resulta em uma impedância cerca de duas vezes maior que aquela obtida para uma conexão no ponto B (ponto central). Neste último caso, a corrente "enxerga" duas impedâncias em paralelo, cada uma de valor próximo àquela referente à conexão em $\mathrm{A}$, desconsiderados os efeitos mútuos. Para a malha, a conexão no ponto central (ponto B) resulta em uma impedância de cerca de metade daquela obtida para uma conexão no ponto A (quina da malha), se desconsiderados os efeitos mútuos.

De uma forma geral, o fator de maior influência na redução da impedância de aterramento é a área de dispersão de corrente, determinada pelo arranjo de eletrodos. Para fenômenos impulsivos, tendo em conta o conceito de comprimento efetivo, tal área fica limitada à região próxima ao ponto de injeção de corrente. Esse aspecto é particularmente relevante para grandes malhas de aterramento e solos mais condutivos. Nesses últimos casos recomenda-se uma distribuição adequada dos pontos de conexão do sis- 

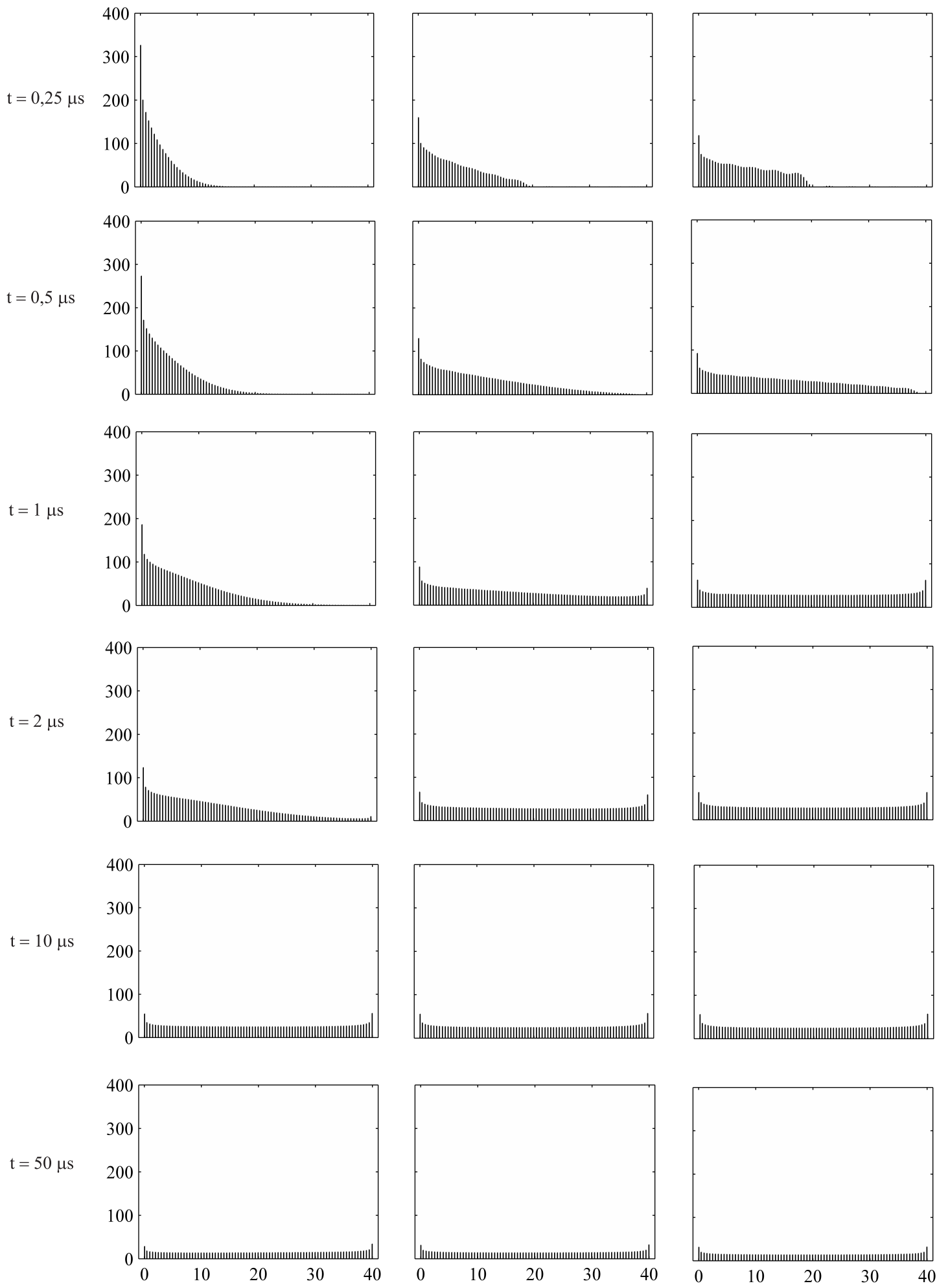

(a) $\rho=100 \Omega . m$

(b) $\rho=500 \Omega . m$

(c) $\rho=1000 \Omega . m$

Figura 7: Distribuição de corrente ao longo do aterramento [corrente dispersa (A) x extensão do eletrodo (m)]. Injeção de um impulso atmosférico 1,2/50 $\mu$ s na extremidade de um eletrodo horizontal de $40 \mathrm{~m}$. 
tema elétrico ao aterramento. Especificamente no caso de malhas de aterramento, a distribuição de pontos de conexão, tendo em conta a área de abrangência para dispersão de corrente, segundo o comprimento efetivo para o solo específico, resulta em reduções significativas da impedância de aterramento.

É importante salientar que os resultados apresentados neste trabalho foram obtidos segundo uma abordagem conservadora, em que os efeitos de ionização do solo e de variação de seus parâmetros elétricos (condutividade $\sigma$ e permissividade $\epsilon)$ com a frequência foram desconsiderados.

A inclusão dos efeitos de ionização do solo conduz a uma redução do valor da impedância impulsiva (Salari, 2006; Visacro, 2007; Salari e Portela, 2008). Todavia, o processo de ionização do solo pouco influi no parâmetro lef, principalmente tendo em conta os valores medianos de amplitude de correntes de descargas atmosféricas (Visacro et alli, 2004) ${ }^{1}$. Apenas para valores muito elevados de corrente espera-se uma ligeira redução no comprimento efetivo, uma vez que, nesses casos, grandes parcelas de corrente são dispersas nas regiões fortemente ionizadas próximas ao ponto de injeção de corrente.

A inclusão da variação dos parâmetros do solo com a frequência, ou seja, o crescimento da condutividade e redução da permissividade com o aumento da frequência de cerca de $100 \mathrm{~Hz}$ até $2 \mathrm{MHz}$, também conduz a uma redução da impedância impulsiva de aterramento (Portela, 1999; Visacro et alli, 2008; Alípio et alli, 2008b e 2009b; Pedrosa et alli 2009; Pedrosa, 2010; Pedrosa et alli 2010a, 2010b e 2010c). Uma vez que a constante de propagação do meio é função direta dos parâmetros eletromagnéticos que o caracteriza, a variação de $\sigma$ e $\epsilon$ com a frequência influi de forma mais acentuada no comprimento efetivo do eletrodo. Em estudo recente, baseado em resultados experimentais do comportamento de $\sigma$ e $\epsilon$ de amostras de solo ao longo do espectro típico de descargas atmosféricas, Alípio et alli (2009b) e Pedrosa (2010) indicam um pequeno aumento do $\ell$ ef, dependendo da resistividade do solo, quando a dependência com a frequência de $\sigma$ e $\epsilon$ é computada. A despeito desse aumento, recomenda-se, novamente, a utilização dos valores de $\ell$ ef previamente determinados, segundo a mesma justificativa

\footnotetext{
${ }^{1} \mathrm{~A}$ maior base de dados de ondas de correntes associadas às descargas atmosféricas corresponde às medições realizadas por Berger na Suíça (Berger, et alli, 1975; Anderson e Eriksson, 1980). No hemisfério sul do planeta, a maior base é a do Morro do Cachimbo (Schroeder, 2001). Os conjuntos de variáveis aleatórias correspondentes aos parâmetros típicos de uma onda de corrente de descarga atmosférica (pico; tempos de frente e de cauda; carga; energia etc.) permitem a definição de grandezas estatísticas de interesse aplicado na engenharia (média; mediana; desvio padrão etc.). Assim sendo, os autores se referem aos valores medianos, por exemplo, das bases de dados da Suíça e de Cachimbo (Brasil) que correspondem, respectivamente, a 30 e $45 \mathrm{kA}$.
}

de que a corrente dispersa para o solo de pontos próximos ao comprimento efetivo é bastante reduzida.

Ainda vale citar que a inclusão de ambos os efeitos comentados anteriormente pode conduzir a valores de coeficiente de impulso inferiores à unidade, principalmente no caso de

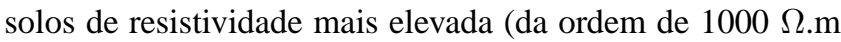
ou superior) Pedrosa (2010). Segundo resultados apresentados por Alípio et alli, (2009b) e Pedrosa (2010), se o comprimento efetivo não é excedido, o coeficiente de impulso é de cerca de 0,75 para um solo de $1000 \Omega$.m ou inferior para solos de resistividade mais elevada. Nesses casos, o aterramento apresenta desempenho impulsivo superior àquele em baixa frequência. Resultados experimentais obtidos por Visacro e Rosado (2009) apontam conclusões neste mesmo sentido.

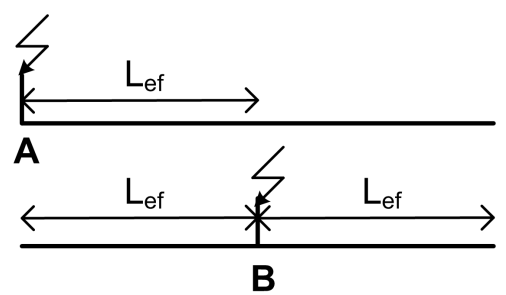

(a)

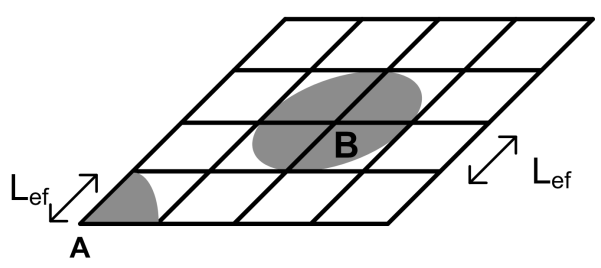

(b)

Figura 8: Influência do ponto de injeção de corrente na impedância de aterramento (Visacro, 2007)

\section{CONCLUSÕES}

Apresentou-se neste trabalho resultados de simulação do comportamento impulsivo de eletrodos de aterramento, com base na aplicação de uma modelagem rigorosa e validada com resultados experimentais. Acredita-se que a partir das análises e comentários enumerados ao longo do texto, este artigo apresenta uma contribuição para a melhor compreensão da resposta do aterramento elétrico frente a correntes de descargas atmosféricas. Algumas das análises apresentadas estão destacadas a seguir:

1. A resposta transitória de eletrodos de aterramentos é definida por dois períodos básicos: 1) período dinâmico (PD) e 2) período permanente (PP). No primeiro período (PD), os efeitos associados a fenômenos de alta frequência, nomeada- 
mente os efeitos reativos e de propagação, são acentuados. Nesse caso, o aterramento é adequadamente representado por uma impedância. No segundo período (PP), os efeitos citados anteriormente são pouco significativos e o comportamento do aterramento é caracterizado pelo parâmetro de baixa frequência resistência de aterramento. A duração do PD é da ordem de até alguns microsegundos e aumenta com o comprimento do eletrodo, condutividade do solo e tempo de frente da onda injetada.

2. O aumento do comprimento do eletrodo implica na redução contínua da resistência de aterramento. Contudo, quando se avalia a resposta impulsiva do aterramento, esse aumento do comprimento pode não refletir diretamente na redução da impedância impulsiva. Atingido um comprimento, chamado comprimento efetivo, posterior aumento de sua extensão se traduz na redução da resistência, mas não da impedância impulsiva de aterramento. O comprimento efetivo depende basicamente do tempo de frente da onda de corrente e da resistividade do solo, sendo tanto maior quanto maior forem essas duas grandezas.

3. A impedância impulsiva de aterramento é igual à resistência de aterramento para eletrodos com extensão até o comprimento efetivo. Além desse comprimento, a impedância é sempre superior a resistência de aterramento. Esse resultado é válido considerando os parâmetros do solo constantes com a frequência.

4. Ao longo do PD, o perfil de corrente que dispersa do eletrodo para o solo é não uniforme com maior concentração da corrente na região próxima ao ponto de injeção de corrente. Nesse período (PD), o comprimento efetivo fornece uma boa referência da extensão do eletrodo que é efetivamente utilizada na dispersão de corrente para o solo. Ao longo do PP todo o eletrodo é efetivamente utilizado para dispersão da corrente. Nesse período (PP), a corrente de dispersão apresenta um perfil tipicamente observado quando o aterramento é submetido a fenômenos de baixa frequência.

5. A resistência de aterramento independe do posicionamento do(s) ponto(s) de injeção de corrente na malha. Por outro lado, a impedância impulsiva pode variar de acordo com os pontos de conexão. Para minimização da impedância de aterramento, os pontos de injeção de corrente na malha devem ser escolhidos observando-se o comprimento efetivo, de acordo com a resistividade do solo em que o aterramento se encontra inserido.

Os autores têm a expectativa de que este trabalho possa ser uma referência para engenheiros da indústria, envolvidos com proteção de sistemas elétricos contra descargas atmosféricas e, também, para aqueles envolvidos com a pesquisa de modelos para predição do comportamento tran- sitório do aterramento e engenheiros projetistas de sistemas elétricos.

\section{REFERÊNCIAS}

Alípio, R. S. (2008a). Modelagem Eletromagnética de Aterramentos Elétricos nos Domínios do Tempo e da Frequência. Dissertação de Mestrado, Programa de Pós-Graduação em Modelagem Matemática e Computacional do Centro Federal de Educação Tecnológica de Minas Gerais, PPGMMC/CEFET-MG, Belo Horizonte - MG.

Alípio, R. S., Schroeder, M. A. O., Afonso, M M. and Oliveira, T. A. S. (2008b). Electromagnetic Fields of Buried Conductors. Proc. of International Conference on Grounding and Earthing (GROUND 2008), Florianópolis, Brazil, pp. 399-402.

Alípio, R. S., Schroeder, M. A. O. and Afonso, M M. (2009a). Computer Analysis of Electromagnetic Transients in Grounding Systems. Proc. of $17^{\text {th }}$ International Conference on the Computation of Electromagnetic Fields (COMPUMAG 2009), Florianópolis, Brazil.

Alípio, R. S., Schroeder, M. A. O., Afonso, M M. and Oliveira, T. A. S. (2009b). The Influence of the Soil Parameters Dependence with Frequency on Impulse Grounding Behavior. Proc. of International Symposium of Lightning Protection (SIPDA 2009), Curitiba, Brazil.

Alípio, R. S., Schroeder, M. A. O., Afonso, M. M. e Oliveira, T. A. S. (2011). Modelagem de Aterramentos Elétricos para Fenômenos de Alta Frequência e Comparação com Resultados Experimentais. Revista Controle \& Automação (SBA), Vol. 22 no. 1, pp. 89102.

Anderson, R. B. and Eriksson, A. J. (1980) Lightning Parameters for Engineering Application. Electra, n $^{\circ}$ 69, pp. 65-102.

Berger, K., Anderson, R. B. and Kröninger, H. (1975). Parameters of Lightning Flashes. Electra, $n^{\circ} 41$, pp. 2337.

Cherchiglia, L. C. L., Bezerra, A. C. G., Markiewicz, R. L., Carmo, L. S., Carvalho A. M. e Amorim, G. E. S. (1997). Otimização de Sistema Computacional para Estimativa do Desempenho de LT's Sob a Ação de Descargas Atmosféricas e sua Aplicação para Melhoria do Desempenho de LT's da CEMIG. VII Encontro Regional Latino-Americano da CIGRÉ, Argentina.

Dawalibi, F and Selby, A. (1993). Electromagnetic Fields of Energized Conductors. IEEE Transactions on Power Delivery, Vol. 8, nº3, pp. 1275-1284. 
Geri, A. (1999). Behavior of Grounding Systems Excited by High Impulse Currents: The Model and its Validation. IEEE Transactions on Power Delivery, Vol. 8, n³, pp. 1008-1017.

Grcev, L. (1996). Computer Analysis of Transients Voltages in Large Grounding Systems. IEEE Transactions on Power Delivery, Vol. 11, ${ }^{\circ}{ }^{2}$, pp. 815-823.

Grcev, L. (2009). Impulse Efficiency of Ground Electrodes. IEEE Transactions on Power Delivery, Vol. 24, $\mathrm{n}^{\mathrm{0}} 1$, pp. 441-451.

Grcev, L. and Dawalibi, F. (1990). An Electromagnetic Model for Transients in Grounding Systems. IEEE Transactions on Power Delivery, Vol. 5, $\mathrm{n}^{\circ} 4$, pp. 1773-1781.

Harrington, R. F. (1993). Field Computation by Moment Methods. IEEE Press, New York.

He, J., Gao, Y., Zeng, R., Zou, J., Liang, X., Zhang, B., Lee, J. and Chang, S. (2005). Effective Length of Counterpoise Wire Under Lightning Current. IEEE Transactions on Power Delivery, Vol. 20, n² 2, pp. 1585-1591.

Liu, Y., Theethayi, N. and Thottappillil, R. (2005). An Engineering Model for Transient Analysis of Grounding System Under Lightning Strikes: Nonuniform Transmission-line Approach. IEEE Transactions on Power Delivery, Vol. 20, $\mathrm{n}^{\mathrm{0}}$ 2, pp. 722-730.

Liu, Y., Zitnik, M. and Thottappillil, R. (2001). An Improved Transmission-line Model of Grounding System. IEEE Transactions on Electromagnetic Compatibility, Vol. 43, n⿳3 3, pp. 348-355.

Lorentzou, M. I., Hatziargyriou, N. D. and Papadias, B. C. (2003). Time Domain Analysis of Grounding Electrodes Impulse Response. IEEE Transactions on Power Delivery, Vol. 18, n² , pp. 517-524.

Mazzetti, C. and Veca, M. G. (1983). Impulse Behavior of Ground Electrodes. IEEE Transactions on Power Apparatus and Systems, Vol. PAS-102, n ${ }^{\circ}$, pp. 3148-3156.

Meliopoulos, A. P. and Moharam, M. G. (1983). Transient Analysis of Grounding Systems. IEEE Transactions on Power Apparatus and Systems, Vol. PAS-102, $\mathrm{n}^{\circ} 2$, pp. 389-399.

Miranda, J. A. (2003). Simulação de Fenômenos Transitórios em Sistemas de Aterramento. Dissertação de Mestrado, Coordenação dos Programas de PósGraduação da Universidade Federal do Rio de Janeiro, COPPE/UFRJ, Rio de Janeiro - RJ.

NBR Coordenação de Isolamento - Procedimento (2000). NBR 6939-2000, Nov. 2000.
Nogueira, R. L. S. (2006). Análise de Sistema de Aterramento sob Solicitações Impulsivas: Otimização e Critérios de Segurança em Aterramentos de Estruturas de Linhas de Transmissão. Dissertação de Mestrado, Coordenação dos Programas de Pós-Graduação da Universidade Federal do Rio de Janeiro, COPPE/UFRJ, Rio de Janeiro - RJ.

Olsen, R. G. and Willis, M. C. (1996). A Comparison of Exact and Quasi-static Methods for Evaluating Grounding Systems at High Frequencies. IEEE Transactions on Power Delivery, Vol. 11, nº2, pp. 1071-1081.

Otero, A. F., Cidrás, J. and del Alamo, J. L. (1999). Frequency-dependent Grounding System Calculation by Means of Conventional Nodal Analysis Technique. IEEE Transactions on Power Delivery, Vol. 8, $\mathrm{n}^{\circ} 3$, pp. 873-878.

Papalexopoulos, A. D. and Meliopoulos, A. P. (1987). Frequency Dependent Characteristics of Grounding Systems. IEEE Transactions on Power Delivery, Vol. 2, n 4 , pp. 1073-1081.

Pedrosa, A. G., Alípio, R. S., Schroeder, M. A. O. e Oliveira R. K. (2009). Estudo comparativo entre Formulações de Medição de Variação da Condutividade e da Permissividade de Solos Típicos com a Frequência. XX Seminário Nacional de Produção e Transmissão de Energia Elétrica (SNPTEE), Recife, Brasil.

Pedrosa, A. G. (2010). Comportamento Transitório de Eletrodos de Aterramento Considerando a Variação da Condutividade e Permissividade de Solos Típicos com a Frequência. Dissertação de Mestrado, Programa de Pós-Graduação em Engenharia Elétrica, Associação Ampla entre a Universidade Federal de São João delRei e o Centro Federal de Educação Tecnológica de Minas Gerais, PPGEL/UFSJ/CEFET-MG, Belo Horizonte - MG.

Pedrosa, A. G., Schroeder, M.A.O., Afonso, M. M. (2010a). Influência dos Parâmetros Eletromagnéticos Dependentes da Frequência de Solos Típicos na Resposta Harmônica de Aterramentos Elétricos. XVIII Congresso Brasileiro de Automática (CBA), Bonito.

Pedrosa, A. G., Schroeder, M.A.O., Afonso, M. M., Oliveira, T. A., Assis, S. C., Braga, A. R. (2010b). Transient Behavior of Horizontal Grounding Electrodes to Impulsive Phenomena Considering Soil Electrical Parameters Frequency-Dependent. International Conference on Grounding and Earthing (GROUND) \& 4th International Conference on Lightning Physics and Effects, Salvador. 
Pedrosa, A. G., Schroeder, M.A.O., Afonso, M. M., Alípio, R. S., Oliveira, T. A., Assis, S. C., Braga, A. R. (2010c). Transient Response of Grounding Electrodes for the Frequency-Dependence of Soil Parameters. IEEE/PES Transmission \& Distribution Latin America, São Paulo.

Portela, C. (1997). Frequency and Transient Behavior of Grounding Systems - I. Physical and Methodological Aspects. Proc. of the IEEE International Symposium on Electromagnetic Compatibility, Austin, U.S.A., pp. 379-384.

Portela, C. (1999). Measurement and Modeling of Soil Electromagnetic Behavior. Proc. of the IEEE International Symposium on Electromagnetic Compatibility, Seattle, U.S.A., pp. 1004-1009.

Ramamoorty, M., Babu Narayanan, M. M., Parameswaran, S. and Mukhedkar, D. (1989). Transient Performance of Grounding Grids. IEEE Transactions on Power Delivery, Vol. 4, $\mathrm{n}^{\mathrm{O}} 4$, pp. 2053-2059.

Rodrigues, M. G. (2004). Metodologia Aproximada para Simulação do Comportamento Transitório de Malhas de Terra e Avaliação da Distribuição de Descargas Atmosféricas. Tese de Doutorado, Coordenação dos Programas de Pós-Graduação da Universidade Federal do Rio de Janeiro, COPPE/UFRJ, Rio de Janeiro - RJ.

Salari, J. C. (2006). Efeito das Descargas Atmosféricas no Desempenho de Linhas de Transmissão - Modelagens nos Domínios do Tempo e da Freqüência. Tese de Doutorado. Coordenação dos Programas de Pós-Graduação de Engenharia da Universidade Federal do Rio de Janeiro, COPPE/UFRJ, Rio de Janeiro - RJ.

Salari, J. C. and Portela, C. (2007). A Methodology for Electromagnetic Transients Calculation - An Application for the Calculation of Lightning Propagation in Transmission Lines. IEEE Transactions on Power Delivery, Vol. 22, $\mathrm{n}^{\circ} 1$, pp. 527-536.

Salari, J. C. and Portela, C. (2008). Grounding systems modeling including soil ionization. IEEE Transactions on Power Delivery, Vol. 23, n 4, pp. 1939-1945.

Schroeder, M. A. O. (2001). Modelo Eletromagnético para Descontaminação de Ondas de Corrente de Descargas Atmosféricas: Aplicação às Medições da Estação do Moro do Cachimbo. Tese de Doutorado. Centro de Pesquisa e Desenvolvimento em Engenharia Elétrica da Universidade Federal de Minas Gerais, CPDEE/UFMG, Belo Horizonte - MG.

Soares, A. (1996). Investigação do Comportamento dos Aterramentos Típicos de Linhas de Transmissão Frente a Descargas Atmosféricas. Dissertação de Mestrado.
Centro de Pesquisa e Desenvolvimento em Engenharia Elétrica da Universidade Federal de Minas Gerais, CPDEE/UFMG, Belo Horizonte - MG.

Velazquez, R. and Mukhedkar, D. (1984). Analytical Modeling of Grounding Electrodes Transient Behavior. IEEE Transactions on Power Apparatus and Systems, Vol. PAS-103, nº, pp. 1314-1322.

Verma, R. and Mukhedkar, D. (1980). Impulse Impedance of Buried Ground Wire. IEEE Transactions on Power Apparatus and Systems, Vol. PAS-99, n 5 , pp. 20032007.

Visacro, S., Soares, A., Schroeder, M. A. O., Cherchiglia, L. C. L. e Carvalho, A. M. (1997). Investigação sobre o Aterramento de Torres do Sistema de Transmissão da CEMIG para Melhoria de Desempenho de Linhas Frente a Descargas Atmosféricas. Seminário Internacional de Proteção Contra Descargas Atmosféricas (SIPDA), São Paulo, Brazil.

Visacro, S. (1992). Modelagem de Aterramentos Elétricos. Tese de Doutorado, Coordenação dos Programas de Pós-Graduação da Universidade Federal do Rio de Janeiro, COPPE/UFRJ, Rio de Janeiro - RJ.

Visacro, S. (2002). Aterramentos Elétricos. Artiliber, São Paulo.

Visacro, S. (2005). Descargas Atmosféricas: Uma Abordagem de Engenharia. Artiliber, São Paulo.

Visacro, S. (2007). A Comprehensive Approach to the Grounding Response to Lightning Currents. IEEE Transactions on Power Delivery, Vol. 22, $\mathrm{n}^{\circ} 1$, pp. 381386.

Visacro, S. and Portela, C. (1992). Modeling of Earthing Sytems for Lightning Protection Applications, Including Propagation Effects. Proc. International Conference on Lightning Protection (ICLP 1992), Germany, pp. 129-132.

Visacro, S. and Soares, A. (2005). HEM: A Model for Simulation of Lightning-Related Engineering Problems. IEEE Transactions on Power Delivery, Vol. 20, $\mathrm{n}^{\circ} 2$, pp. 1206-1207.

Visacro, S., Pinto, W. L. F., Almeida, F. S., Murta, M. H. and Rosado, G. (2008). Experimental evaluation of soil parameter behavior in the frequency range associated to lightning currents. Proc. 29th International Conference on Lightning Protection (ICLP 2008), Sweden, paper $5 \mathrm{c}-2$. 
Visacro, S., Soares, A., Schroeder, M. A. O., Cherchiglia, L. C. L. and Sousa, V. J. (2004). Statistical Analysis of Lightning Current Parameters: Measurements at Morro do Cachimbo Station. Journal on geophysical research, Vol. 109, D01105, pp. 1-11.

Visacro, S., Rosado G. (2009). Response to Grounding Electrodes to Impulsive Currents: An Experimental Evaluation. IEEE Transactions on Electromagnetic Compatibility, Vol. 51, nº 1, pp. 161-164.

Zeng, R., Gong, X., He, J., Zhang, B. and Gao, Y. (2008). Lightning Impulse Performance of Grounding Grids for Substation Considering Soil Ionization. IEEE Transactions on Power Delivery, Vol. 22, nº 2, pp. 667-677. 